UG-00-11

OU-HET 362

DAMTP-2000-103

hep-th/0009147

\title{
M-BRANE INTERPOLATIONS AND $(2,0)$ RENORMALIZATION GROUP FLOW
}

\author{
Eric Bergshoeff*, ${ }^{*}$, Rong-Gen Cai ${ }^{\sharp, 2}$, \\ Nobuyoshi Ohta ${ }^{\sharp, 3}$ and Paul K. Townsend ${ }^{\dagger, 4}$ \\ * Institute for Theoretical Physics, University of Groningen, \\ Nijenborgh 4, 9747 AG Groningen, The Netherlands \\ \# Department of Physics, Osaka University, \\ Toyonaka, Osaka 560-0043, Japan \\ $\dagger$ DAMTP, University of Cambridge, \\ Centre for Mathematical Sciences, Wilberforce Road, \\ Cambridge CB3 0WA, UK
}

\begin{abstract}
We obtain the M5-M2-MW bound state solutions of 11-dimensional supergravity corresponding to the $1 / 2$ supersymmetric vacua of the M5-brane equations with constant background fields. In the 'near-horizon' case the solution interpolates between the $a d S_{7} \times S^{4}$ Kaluza-Klein vacuum and $\mathrm{D}=11$ Minkowski spacetime via a Domain Wall spacetime. We discuss implications for renormalization group flow of $(2,0) \mathrm{D}=6$ field theories.
\end{abstract}

\footnotetext{
${ }^{1}$ E-mail: bergshoe@phys.rug.nl

${ }^{2}$ E-mail: cai@het.phys.sci.osaka-u.ac.jp

${ }^{3}$ E-mail: ohta@phys.sci.osaka-u.ac.jp

${ }^{4}$ E-mail: p.k.townsend@damtp.cam.ac.uk
} 


\section{Introduction}

Following many studies of D-branes in constant $B$-field backgrounds, a number of papers have considered the analogous problem of the M5-brane in a constant background 3-form gauge field $C$ (e.g. [1]). The background field $C$ appears, via its pullback, in the M5-brane action through the worldvolume 3 -form field strength $H=d A-C$. An M5-brane in a constant $C$ background is therefore equivalent to an M5-brane in the M-theory vacuum but with constant worldvolume 3 -form $H$. There is a class of such constant M5-brane configurations that preserve all 16 supersymmetries of the M5-brane vacuum [2]. This class is characterized by the M5-brane charge $y$, the M-Wave (MW) charge p, which is a momentum in one direction in the M5-brane, and the skew eigenvalues $\xi_{1}, \xi_{2}$ of the spatial components of $H$ in the 4-directions orthogonal to $p$; these skew eigenvalues are M2-brane charges, as one discovers by a computation of the supersymmetry algebra of the M5-brane Noether charges [2]. This calculation leads to the conclusion, for a particular choice of worldvolume coordinates and assuming that the tension is normalized to unity, that all 16 worldvolume supersymmetries will be preserved if and only if the equation

$$
\left(\xi_{1} \Gamma_{012}+\xi_{2} \Gamma_{034}+p \Gamma_{05}+y \Gamma_{012345}\right) \epsilon=\epsilon
$$

admits 16 linearly-independent non-zero solutions for the constant real $\mathrm{D}=11$ spinor $\epsilon$. This is the case if and only if the charges $\left(y, p, \xi_{1}, \xi_{2}\right)$ satisfy

$$
\begin{array}{r}
\xi_{1}^{2}+\xi_{2}^{2}+p^{2}+y^{2}=1, \\
\xi_{1} \xi_{2}-p y=0 .
\end{array}
$$

The solutions of these constraints can be parametrized by two angles $\left(\theta_{1}, \theta_{2}\right)$ as follows:

$$
\begin{aligned}
\xi_{1} & =s_{1} c_{2}, \quad \xi_{2}=c_{1} s_{2}, \\
p & =s_{1} s_{2}, \quad y=c_{1} c_{2} .
\end{aligned}
$$

where

$$
s_{i}=\sin \theta_{i}, \quad c_{i}=\cos \theta_{i}, \quad(i=1,2) .
$$

Thus, there is a two-parameter family of M5-brane vacua. They are vacua in the sense that they preserve all 16 supersymmetries, although they differ in energy because they minimise the energy subject to different boundary conditions (namely that the fields approach their prescribed constant values at infinity).

The above result was found from the action of a single M5-brane. The action for multiple M5-branes is not known but the low energy dynamics is presumed to be governed by a $(2,0)$-supersymmetric $\mathrm{D}=6$ superconfomal field theory (see e.g. [3]). It is thus natural to suppose that this theory is a particular, superconformal, member of a 2-parameter family of $(2,0)$ field theories, parameterized as above. The superconformal $(2,0)$ theory is believed to be equivalent, via the adS/CFT correspondence, to M-theory on $a d S_{7} \times S^{4}$, which is the 'near-horizon' limit of the $\mathrm{D}=11$ supergravity M5-brane. The other, nonconformal, $(2,0)$ field theories are then presumably equivalent to M-theory in a background that is the near-horizon limit of a bound state solution of an M5-brane with M2-branes and an M-Wave, the charges of these constituents being related in the way described above. We thus expect there to exist a family of $1 / 2$ supersymmetric M5-M2-MW bound state solutions of $\mathrm{D}=11$ supergravity parameterized by the two angles $\left(\theta_{1}, \theta_{2}\right)$. One purpose of this paper is to present this family of solutions, which we obtain using the methods of [4, 5]. The solutions are essentially the lift to $\mathrm{D}=11$ of $\mathrm{D}$-brane bound state solutions found in [4. 6]. Each depends on a single harmonic function $H$ on $\mathbb{E}^{5}$, the space transverse to the M5-brane's worldvolume in eleven dimensions. The full stationary $\mathrm{D}=11$ solution 
has not previously been given, although the static M5-M2-brane bound state solutions were found in [7] and the M2-MW case is the boosted M2-brane of [8].

We shall be interested here in the 'near-horizon' case obtained by choosing

$$
H=1 / r^{3}
$$

where $r$ is the radial distance from the brane in the transverse $\mathbb{E}^{5}$ space. For this choice, the pure M5-brane solution becomes the $a d S_{7} \times S^{4}$ Kaluza-Klein vacuum 9, with isometry group

$$
S O(2,6) \times S O(5)
$$

as expected by the equivalence of M-theory in this background to the conformal $(2,0)$ theory. In the generic M5-M2-MW case the isometry group is

$$
\mathbb{R}^{2} \times I S O(2) \times I S O(2) \times S O(5)
$$

but this is typically enhanced in either of the limits $r \rightarrow 0$ or $r \rightarrow \infty$. In particular, it is always enhanced to $S O(2,6) \times S O(5)$ in the $r \rightarrow 0$ limit, provided that the M5 charge is non-zero; this generalizes the observation of [7] that the M5-brane dominates the M5-M2 solution in this limit. For the pure M5-brane the $r \rightarrow \infty$ limit yields the same as the $r \rightarrow 0$ limit (for $H=1 / r^{3}$ ). This was to be expected from the conjectured equivalence of M-theory in the near-horizon M5-brane background with the superconformal $(2,0)$ theory. In all other cases the $r \rightarrow \infty$ limit yields an asymptotic spacetime that is not $a d S_{7} \times S^{4}$. We interpret this to mean that the non-conformal $(2,0)$ theories flow to the conformal $(2,0)$ theory in the IR limit.

Of particular interest is a limit in the two-parameter space corresponding to a critical electric component of $H$, as this has been argued to lead to an Open Membrane (OM) theory [10, 11]. Because of the non-linear self-duality condition obeyed by $H$, this limit corresponds to one in which some magnetic components of $H$ go to infinity. This implies that the M5-brane tension must also go to infinity, and if one rescales to keep the tension at unity then the limit is one in which either $\xi_{1}$ or $\xi_{2}$ becomes large relative to $y$. The only way the relations (2) can be satisfied in this limit is if either $\xi_{1} \rightarrow 1$ or $\xi_{2} \rightarrow 1$ (but not both). The supergravity dual in this limit was studied in [12] for the special case in which $\xi_{2}=p=0$, using a form of the static M5-M2 bound state solution of $\mathrm{D}=11$ supergravity found in [13]. An asymptotic 'smeared membrane' spacetime was found and argued to be the background associated with the supergravity dual to OM-theory.

Part of the motivation for the work reported here was to get a better understanding of the renormalization group (RG) flow to the conformal $(2,0)$ theory by considering the general M5-M2-MW solution and its interpolation properties. For the special case of the M5-M2 bound state we find an interpolation between the $a d S_{7} \times S^{4}$ vacuum (for $r \rightarrow 0$ ) and (for $r \rightarrow \infty$ ) the near-horizon limit of the M2-brane as a solution of the maximal $\mathrm{D}=8$ supergravity [14], for which the 'dual-frame' 8 -metric is $a d S_{4} \times S^{4}$. This solution was first obtained as a $\mathrm{D}=4$ domain wall $(\mathrm{DW})$ solution of $\left(T^{3} \times S^{4}\right)$-compactified $\mathrm{D}=11$ supergravity [15], so we shall refer to it as the DW solution. The generic M5-M2-MW solution, however, has quite different interpolation properties. When the MW charge is non-zero the metric is asymptotic, as $r \rightarrow \infty$, to a flat $\mathrm{D}=11$ vacuum spacetime. This is achieved via an intermediate DW spacetime.

We begin our presentation of these results with the construction of the general $1 / 2$ supersymmetric M5-M2-MW solution of $\mathrm{D}=11$ supergravity, which we obtain by a series of solution-generating manipulations from the $\mathrm{D} 2$-brane solution of IIA $\mathrm{D}=10$ supergravity. We then specialize to the 'near-horizon' choice (5) of harmonic function, and consider the $r \rightarrow 0$ and $r \rightarrow \infty$ limits. We conclude with a summary of the RG interpretation and a discussion of some related issues. 


\section{Construction}

We start from the $\mathrm{D} 2$-brane solution of $\mathrm{D}=10$ IIA supergravity

$$
\begin{aligned}
d s_{A}^{2}= & H^{-1 / 2}\left(-d t^{2}+d x_{1}^{2}+d x_{3}^{2}\right)+H^{1 / 2}\left(d x_{2}^{2}+d x_{4}^{2}+d r^{2}+r^{2} d \Omega_{4}^{2}\right), \\
& \phi=\frac{1}{4} \log H, \quad C=\frac{1-H}{H} d t \wedge d x_{1} \wedge d x_{3},
\end{aligned}
$$

where $H$ is a harmonic function on the transverse space, which we shall take to be independent of $x_{2}$ and $x_{4}$; in other words, we have a D2-brane 'smeared' in the $x_{2}$ and $x_{4}$ directions, which we assume are compact. We now define new rotated coordinates $\left(\tilde{x}_{1}, \tilde{x}_{2}, \tilde{x}_{3}, \tilde{x}_{4}\right)$ by

$$
\left(\begin{array}{l}
x_{1} \\
x_{2}
\end{array}\right)=\left(\begin{array}{cc}
c_{1} & -s_{1} \\
s_{1} & c_{1}
\end{array}\right)\left(\begin{array}{l}
\tilde{x}_{1} \\
\tilde{x}_{2}
\end{array}\right), \quad\left(\begin{array}{l}
x_{3} \\
x_{4}
\end{array}\right)=\left(\begin{array}{cc}
c_{2} & -s_{2} \\
s_{2} & c_{2}
\end{array}\right)\left(\begin{array}{l}
\tilde{x}_{3} \\
\tilde{x}_{4}
\end{array}\right)
$$

where $s_{i}$ and $c_{i}$ are the sines and cosines of (ब). In the new coordinates the IIA solution (8) is

$$
\begin{aligned}
& d s_{A}^{2}=-H^{-1 / 2} d t^{2}+H^{-1 / 2}\left(c_{1}^{2}+H s_{1}^{2}\right) d x_{1}^{2}+H^{-1 / 2}\left(s_{1}^{2}+H c_{1}^{2}\right) d x_{2}^{2} \\
&+2 H^{-1 / 2}(H-1) c_{1} s_{1} d x_{1} d x_{2}+H^{-1 / 2}\left(c_{2}^{2}+H s_{2}^{2}\right) d x_{3}^{2} \\
&+H^{-1 / 2}\left(s_{2}^{2}+H c_{2}^{2}\right) d x_{4}^{2}+2 H^{-1 / 2}(H-1) c_{2} s_{2} d x_{3} d x_{4} \\
&+H^{1 / 2}\left[d r^{2}+r^{2} d \Omega_{4}^{2}\right], \\
& \phi=\frac{1}{4} \log H, \quad C=\frac{1-H}{H} d t \wedge\left(d x_{1} c_{1}-d x_{2} s_{1}\right) \wedge\left(d x_{3} c_{2}-d x_{4} s_{2}\right),
\end{aligned}
$$

where we have now dropped the tildes.

Performing a T-duality in the $x_{2}$-direction, we obtain the IIB supergravity solution

$$
\begin{aligned}
d s_{B}^{2}= & -H^{-1 / 2} d t^{2}+\frac{H^{1 / 2}}{E_{1}}\left(d x_{1}^{2}+d x_{2}^{2}\right)+H^{-1 / 2}\left(c_{2}^{2}+H s_{2}^{2}\right) d x_{3}^{2} \\
& +H^{-1 / 2}\left(s_{2}^{2}+H c_{2}^{2}\right) d x_{4}^{2}+2 H^{-1 / 2}(H-1) c_{2} s_{2} d x_{3} d x_{4} \\
& +H^{1 / 2}\left[d r^{2}+r^{2} d \Omega_{4}^{2}\right], \\
\varphi= & \frac{1}{2} \log \frac{H}{E_{1}} \\
D= & \frac{1-H}{E_{1}} c_{1} d t \wedge d x_{1} \wedge d x_{2} \wedge\left(d x_{3} c_{2}-d x_{4} s_{2}\right), \\
B^{(1)}= & \frac{H-1}{E_{1}} c_{1} s_{1} d x_{1} \wedge d x_{2}, \\
B^{(2)}= & \frac{1-H}{H} d t \wedge\left(d x_{3} c_{2}-d x_{4} s_{2}\right) s_{1},
\end{aligned}
$$

and a further T-duality in the $x_{4}$-direction converts this to the IIA supergravity solution

$$
\begin{aligned}
d s_{A}^{2} & =H^{1 / 2}\left[-H^{-1} d t^{2}+\frac{1}{E_{1}}\left(d x_{1}^{2}+d x_{2}^{2}\right)+\frac{1}{E_{2}}\left(d x_{3}^{2}+d x_{4}^{2}\right)+d r^{2}+r^{2} d \Omega_{4}^{2}\right], \\
\phi & =\log H^{3 / 4} E_{1}^{-1 / 2} E_{2}^{-1 / 2}, \\
A & =\frac{1-H}{H} s_{1} s_{2} d t, \\
B & =\frac{H-1}{E_{1}} c_{1} s_{1} d x_{1} \wedge d x_{2}+\frac{H-1}{E_{2}} c_{2} s_{2} d x_{3} \wedge d x_{4}, \\
d C & =d\left(\frac{1-H}{E_{1}}\right) c_{1} s_{2} \wedge d t \wedge d x_{1} \wedge d x_{2}+d\left(\frac{1-H}{E_{2}}\right) c_{2} s_{1} \wedge d t \wedge d x_{3} \wedge d x_{4}-c_{1} c_{2} \star d H,
\end{aligned}
$$


where

$$
E_{1}=s_{1}^{2}+H c_{1}^{2}, \quad E_{2}=s_{2}^{2}+H c_{2}^{2} .
$$

This is the desired D0-D2-D2-D4 brane solution [4, 6 .

Uplifting to 11 dimensions, we get the following new $1 / 2$ supersymmetric solution of $\mathrm{D}=11$ supergravity :

$$
\begin{aligned}
& d s_{11}^{2}=\left(E_{1} E_{2}\right)^{1 / 3}\left[-H^{-1}\left[1-(1-H)^{2} E_{1}^{-1} E_{2}^{-1} s_{1}^{2} s_{2}^{2}\right] d t^{2}\right. \\
&+2 E_{1}^{-1} E_{2}^{-1}(1-H) s_{1} s_{2} d t d x_{\natural}+H E_{1}^{-1} E_{2}^{-1} d x_{\natural}^{2} \\
&\left.+E_{1}^{-1}\left(d x_{1}^{2}+d x_{2}^{2}\right)+E_{2}^{-1}\left(d x_{3}^{2}+d x_{4}^{2}\right)+d r^{2}+r^{2} d \Omega_{4}^{2}\right], \\
& d C= d\left(\frac{1-H}{E_{1}}\right) c_{1} s_{2} \wedge d t \wedge d x_{1} \wedge d x_{2}+d\left(\frac{1-H}{E_{2}}\right) c_{2} s_{1} \wedge d t \wedge d x_{3} \wedge d x_{4} \\
&+d\left(\frac{H-1}{E_{1}}\right) c_{1} s_{1} \wedge d x_{1} \wedge d x_{2} \wedge d x_{\natural}+d\left(\frac{H-1}{E_{2}}\right) c_{2} s_{2} \wedge d x_{3} \wedge d x_{4} \wedge d x_{\natural} \\
& \quad-c_{1} c_{2} \star d H,
\end{aligned}
$$

When $s_{1} s_{2}=0$ this reduces to the M5-M2 brane solution of [7]; the subcase with $s_{1}=$ $s_{2}=0$ is the pure M5-brane. When $c_{1} c_{2}=0$ it is the boosted membrane solution of [8]; the subcase with $c_{1}=c_{2}=0$ is the pure M-wave solution. The general case is a bound state solution of an M5-brane with an M-Wave and two orthogonal M2-branes. It has the isometry group ( $(7)$; the $\mathbb{R}^{2}$ factor is generated by the Killing vector fields $\partial / \partial t$ and $\partial / \partial x_{\natural}$. Although $\partial / \partial t$ is not timelike for all $r$, the Killing vector field

$$
k=\frac{\partial}{\partial t}+s_{1} s_{2} \frac{\partial}{\partial x_{\natural}},
$$

is. For this reason it is convenient to define a new space coordinate $\tilde{x}$ by

$$
\tilde{x}=x_{\natural}-s_{1} s_{2} t .
$$

In the new coordinates $k=\partial / \partial t$. The metric is

$$
\begin{aligned}
d s^{2}= & \left(E_{1} E_{2}\right)^{-\frac{2}{3}}\left\{-\left(H c_{1}^{2} c_{2}^{2}+c_{1}^{2} s_{2}^{2}+c_{2}^{2} s_{1}^{2}\right) d t^{2}+2 s_{1} s_{2} d t d \tilde{x}+H d \tilde{x}^{2}\right. \\
& \left.+E_{2}\left(d x_{1}^{2}+d x_{2}^{2}\right)+E_{1}\left(d x_{3}^{2}+d x_{4}^{2}\right)+E_{1} E_{2}\left(d r^{2}+r^{2} d \Omega_{4}^{2}\right)\right\},
\end{aligned}
$$

and the 4 -form field strength is

$$
\begin{aligned}
F=- & d H \wedge\left[c_{1} E_{1}^{-2}\left(s_{2} c_{1}^{2} d t-s_{1} d \tilde{x}\right) d x_{1} \wedge d x_{2}+c_{2} E_{2}^{-2}\left(s_{1} c_{2}^{2} d t-s_{2} d \tilde{x}\right) d x_{3} \wedge d x_{4}\right] \\
& -c_{1} c_{2} \star d H
\end{aligned}
$$

This will be the starting point for the analysis to follow.

\section{Interpolations}

A simple choice of the harmonic function $H$ in the M5-M2-MW solution is $H=a+1 / r^{3}$ for non-negative constant $a$. When $a>0$ the solution is asymptotically flat. Here we shall be interested in the behaviour of the 'near-horizon' solution with $a=0$; that is, with $H=1 / r^{3}$. 
We begin by examining the behaviour as $r \rightarrow 0$. Provided $c_{1} c_{2}$ is non-zero (i.e. nonzero M5-charge) we find the asymptotic solution

$$
\begin{aligned}
d s^{2}= & \left(c_{1} c_{2}\right)^{\frac{2}{3}}\left\{r\left[-d t^{2}+\left(c_{1} c_{2}\right)^{-2} d \tilde{x}^{2}+c_{2}^{-2}\left(d x_{1}^{2}+d x_{2}^{2}\right)+c_{1}^{-2}\left(d x_{3}^{2}+d x_{4}^{2}\right)\right]\right. \\
& \left.+r^{-2} d r^{2}+d \Omega_{4}^{2}\right\}, \\
F= & 3 c_{1} c_{2} r^{-4} \star d r,
\end{aligned}
$$

which is the $a d S_{7} \times S^{4}$ Kaluza-Klein vacuum. This is exactly the same as the near-horizon limit of the pure M5-brane solution [9]. Thus the M5-brane 'dominates' as $r \rightarrow 0$. This result was found previously for the static M5-M2 solution in [7]; we now see that it is true for the general stationary M5-M2-MW solution.

In the special case that the M5-brane charge vanishes $\left(c_{1} c_{2}=0\right)$ it is the M2-brane which dominates in the $r \rightarrow 0$ limit. To see this we set $c_{2}=0$, in which case

$$
E_{2}=1, \quad E_{1}=E \equiv s^{2}+H c^{2},
$$

and the solution (17) reduces to

$$
\begin{aligned}
d s^{2} & =E^{-\frac{2}{3}}\left\{-c^{2} d t^{2}+2 s d t d \tilde{x}+H d \tilde{x}^{2}+d x_{1}^{2}+d x_{2}^{2}+E\left[d x_{3}^{2}+d x_{4}^{2}+d r^{2}+r^{2} d \Omega_{4}^{2}\right]\right\} \\
F & =-c E^{-2} d H \wedge\left\{c^{2} d t-s d \tilde{x}\right\} \wedge d x_{1} \wedge d x_{2} .
\end{aligned}
$$

In the limit $r \rightarrow 0$ this becomes

$$
\begin{aligned}
d s^{2} \sim & c^{-4 / 3} r\left\{r\left[-c^{2} d \tau^{2}+d x_{1}^{2}+d x_{2}^{2}\right]+c^{2} r^{-2} d r^{2}+c^{2} d \Omega_{4}^{2}\right\} \\
& +c^{-4 / 3} r^{-1}\left[d \tilde{x}^{2}+c^{2}\left(d x_{3}^{2}+d x_{4}^{2}\right)\right] \\
F \sim & 3 c^{-1} r^{2} d r \wedge d \tau \wedge d x_{1} \wedge d x_{2},
\end{aligned}
$$

where

$$
\tau=t-\frac{s}{c^{2}} \tilde{x} .
$$

This is just the near-horizon limit of the M2-brane, as a solution of the $T^{3}$-compactified $\mathrm{D}=11$ supergravity; the 8-metric in the curly parenthesis is the 'dual-frame' ad $S_{4} \times S^{4}$ 8 -metric [14]. In summary, there is a 'dominance' hierarchy among the M5, M2 and MW components in the $r \rightarrow 0$ limit with the M5 dominating the M2 and MW and the M2 dominating the MW. It follows that the M5-brane dominates the M-Wave, which means that the singularity of the pure M-Wave solution is removed when it is part of the generic M5-M2-MW solution.

We now turn to the $r \rightarrow \infty$ limit. We shall begin with the static M5-M2 case by setting $s_{2}=0$. In this case

$$
E_{1}=E \equiv s^{2}+H c^{2}, \quad E_{2}=H,
$$

and, assuming that $s$ is non-zero, the asymptotic solution is

$$
\begin{aligned}
d s^{2} & =s^{\frac{2}{3}} r\left\{r\left[-d t^{2}+d x_{3}^{2}+d x_{4}^{2}\right]+r^{-2} d r^{2}+d \Omega_{4}^{2}+s^{-2} r^{-2}\left[d \tilde{x}^{2}+d x_{1}^{2}+d x_{2}^{2}\right]\right\} \\
F & \sim 3 s r^{2} d r \wedge d t \wedge d x_{3} \wedge d x_{4} .
\end{aligned}
$$

Remarkably, this is the same (after some trivial rescaling of coordinates) as the solution found above in the $r \rightarrow 0$ limit of the M2/MW solution. This result depends crucially on $s \neq 0$ (and, of course, on $a=0$ ). When $s=0$, we have the pure M5-brane solution for which the $r \rightarrow \infty$ and $r \rightarrow 0$ limits are identical. The 'mixed' M5-M2 case is thus quite 
different. We shall discuss the significance of this below, but here we may remark that it implies a 'dominance' of the M2 over the M5 in the $r \rightarrow \infty$ limit.

We now turn to the $r \rightarrow \infty$ limit of the generic stationary solution with non-zero $s_{1} s_{2}$. Defining

$$
\tau=t-\left(s_{1} s_{2} A\right)^{-1} \tilde{x}, \quad A=\frac{c_{1}^{2}}{s_{1}^{2}}+\frac{c_{2}^{2}}{s_{2}^{2}},
$$

we find in this case that

$$
\begin{aligned}
d s^{2} & \sim\left(s_{1} s_{2}\right)^{\frac{2}{3}}\left\{-A d \tau^{2}+\frac{1}{A s_{1}^{2} s_{2}^{2}} d \tilde{x}^{2}+\frac{1}{s_{1}^{2}}\left(d x_{1}^{2}+d x_{2}^{2}\right)+\frac{1}{s_{2}^{2}}\left(d x_{3}^{2}+d x_{4}^{2}\right)+d r^{2}+r^{2} d \Omega_{4}^{2}\right\} \\
F & \sim 0 .
\end{aligned}
$$

This is a flat vacuum solution of $\mathrm{D}=11$ supergravity, which is remarkable given that we are discussing the 'near-horizon' solution with $a=0$ ! This result can be interpreted as a dominance of the M-Wave over either the M2-brane or the M5-brane in the $r \rightarrow \infty$ limit because the static M2/M5 solution is not asymptotically flat when $a=0$ whereas the M-wave is. Thus, the dominance hierarchy for $r \rightarrow 0$ is precisely reversed when $r \rightarrow \infty$.

\section{Discussion}

We have now discussed both the $r \rightarrow 0$ limit and the $r \rightarrow \infty$ limits in both the generic case, and all special cases, assuming that $a=0$, i.e. that $H=1 / r^{3}$. The various special cases that arise can be understood as particular features of the generic solution in some characteristic range of the radial coordinate $r$. Suppose that all charges $\xi_{1}, \xi_{2}, y, p$ are non-zero but that one membrane charge is much larger than the other three charges; this corresponds to the critical limit of constant $H$ on the M5-brane. In this case we expect the solution to look like that of the $\left(a=0\right.$ and $T^{3}$-compactified) M2-brane for $r$ not too small or large, i.e. the DW solution. However, for sufficiently small $r$ the M5-brane will dominate and the solution must approach the $a d S_{7} \times S^{4} \mathrm{KK}$ vacuum. On the other hand, for sufficiently large $r$ the MW will dominate and the solution must go to the flat $\mathrm{D}=11$ vacuum. Thus, the dominance hierarchy translates to a sequential interpolation from this flat $\mathrm{D}=11$ vacuum at $r \approx \infty$ to the $\mathrm{DW}$ spacetime at $r \sim 1$ and then on to the $a d S_{7} \times S^{4} \mathrm{KK}$ vacuum at $r \approx 0$. This sequential interpolation corresponds to RG flow from some 11-dimensional theory in the extreme UV (presumably M-theory) to the $(2,0) \mathrm{D}=6 \mathrm{SCFT}$ in extreme IR, passing through some intermediate theory which, by the QFT/DW correspondence [14], is presumably some $\mathrm{D}=3$ field theory on the $\mathrm{D}=4$ Domain Wall.

For the special case of an M2-MW bound state with zero M5 charge, the dimension of the transverse space jumps from 5 to 7 . We can then choose $H$ to be a harmonic function on this 7 -space, and the simplest choice is $H=1 / \rho^{5}$, where $\rho$ is the radial distance from the origin of $\mathbb{E}^{7}$. For the pure M2-brane this yields the $a d S_{4} \times S^{6} \times S^{1}$ DW solution discussed in [14] as the near-horizon limit of the IIA D2-brane. This is also the asymptotic spacetime as $r \rightarrow 0$ in the 'mixed' M2-MW case, but in that case the $r \rightarrow \infty$ limit yields a flat $\mathrm{D}=11$ vacuum. This may correspond to RG flow from the $\mathrm{D}=11$ theory to a non-conformal $\mathrm{D}=3$ field theory on the $\mathrm{D} 2$-brane, but in this case one expects the extreme IR limit to be a conformal $\mathrm{D}=3$ field theory on the M2-brane, dual to the $a d S_{4} \times S^{7}$ vacuum. This suggests that the choice $H=1 / \rho^{5}$ of the harmonic function is special, and that it could be replaced by a more general harmonic function on $\mathbb{E}^{7} \times S^{1}$. Note that no analogous issue arises when the M5-brane charge is non-zero because whereas the M-Wave direction is orthogonal to the M2-brane it is parallel to the M5-brane. 
Acknowledgements: NO and PKT thank the University of Tokyo for hospitality at the SI2000 Fujiyama workshop, where this work was initiated. EB thanks DAMTP for hospitality. The work of RGC and NO was supported in part by Grants-in-Aid for Scientific Research Nos. 99020, 12640270 and Grant-in-Aid on the Priority Area: Supersymmetry and Unified Theory of Elementary Particles.

\section{References}

[1] N. Seiberg and E. Witten String theory and non-commutative geometry, JHEP 9909 (1999) 032;

M. Alishahiha, Y. Oz and J.G. Russo, Supergravity and light-like non-commutativity, JHEP 0009 (2000) 002;

D. Lüst and A. Miemiec, Supersymmetric M5-branes with $H$ field, Phys. Lett. 476B (2000) 395;

E. Bergshoeff, D. Berman, J.P. van der Schaar and P. Sundell, A noncommutative M-theory five-brane, hep-th/0005026;

S. Kawamoto and N. Sasakura, Open membranes in a constant $C$-field background and noncommutative boundary strings, JHEP 0007 (2000) 014.

[2] D. Sorokin and P.K. Townsend, M-theory superalgebra from the M-5-brane, Phys. Lett. 412B (1997) 265.

[3] N. Seiberg and E. Witten, Comments on string dynamics in six dimensions, Nucl. Phys. B471 (1996) 121.

[4] J.C. Breckenridge, G. Michaud and R.C. Myers, More D-brane bound states, Phys. Rev. D55 (1997) 6438.

[5] M. S. Costa and G. Papadopoulos, Superstring dualities and p-brane bound states, Nucl. Phys. B510 (1998) 217.

[6] T. Harmark and N.A. Obers, Phase structure of non-commutative field theories and spinning brane bound states, JHEP 0003 (2000) 024.

[7] J.M. Izquierdo, N.D. Lambert, G. Papadopoulos and P.K. Townsend, Dyonic Membranes, Nucl. Phys. B460 (1996) 560.

[8] J. Russo and A. Tseytlin, Waves, boosted branes and BPS states in M theory, Nucl. Phys. B90 (1997) 121.

[9] G.W. Gibbons and P.K. Townsend, Vacuum interpolation in supergravity via super p-branes, Phys. Rev. Lett. 71 (1993) 3754.

[10] R. Gopakumar, S. Minwalla, N. Seiberg and A. Strominger, (OM) Theory in diverse dimensions, JHEP 0008 (2000) 008.

[11] E. Bergshoeff, D. Berman and P. Sundell, Critical fields on the M5-brane and noncommutative open strings, hep-th/0006112.

[12] D.S. Berman and P. Sundell, Flowing to a non-commutative (OM) five brane via its supergravity dual, hep-th/0007052.

[13] M. Cederwall, U. Gran, M. Holm and B.E.W. Nilsson, Finite tensor deformations of supergravity solitons, JHEP 9902 (1999) 003. 
[14] H.J. Boonstra, K. Skenderis and P.K. Townsend, The domain-wall/QFT correspondence, JHEP 9901 (1999) 003.

[15] P.M. Cowdall, On gauged maximal supergravity in six dimensions, JHEP 9906 (1999) 018. 